\title{
Form-Specific Explicit and Implicit Memory in the Right Cerebral Hemisphere
}

\author{
Chad J. Marsolek, Larry R. Squire, Stephen M. Kosslyn, and Michael E. Lulenski
}

\begin{abstract}
Results from 2 divided visual field (DVF) experiments indicate that in some conditions both explicit and implicit memory are greater when same-letter-case stimuli are presented directly to the right cerebral hemisphere (in the left visual field) than when they are presented directly to the left (in the right visual field). Explicit memory was measured with word-stem cued recall, and implicit memory was measured with word-stem completion priming. Words were presented centrally during encoding, and word stems were presented directly to the right hemisphere or to the left hemisphere during testing. Results for explicit memory contrast with findings from a previous DVF study that used a different procedure, those for implicit memory replicate previous DVF findings, and both results corroborate positron emission tomography findings. We suggest that a formspecific system in the right hemisphere may contribute to both explicit and implicit memory.
\end{abstract}

Research that uses positron emission tomography (PET) is currently helping to delineate areas of the human brain that play specific roles in reading and remembering words (for a review, see Petersen \& Fiez, 1993). One recent discovery from PET is that a region of posterior cortex in the right cerebral hemisphere implements at least some of the processing that underlies visual repetition priming of words (Squire et al., 1992). This discovery complements recent findings from divided visual field (DVF) experiments. Under certain conditions, visual repetition priming of words is greater when test stimuli are presented directly to the right hemisphere (in the left visual field) than directly to the left hemisphere (in the right visual field; Marsolek, Kosslyn, \& Squire, 1992). A second important finding from PET is that processing in the hippocampal region of the right hemisphere is involved in visual cued recall of words (Squire et al., 1992). However, findings from the DVF experiments do not corroborate this PET result. At least

Chad J. Marsolek and Michael E. Lulenski, Department of Psychology, University of Arizona; Larry R. Squire, Department of Veterans Affairs Medical Center, San Diego, and Departments of Psychiatry and Neurosciences, University of California, San Diego; Stephen M. Kosslyn, Department of Psychology, Harvard University.

The research was supported by a grant from the McDonnell-Pew Cognitive Neuroscience Program, the Arizona Cognitive Science Program, the Medical Research Service of the Department of Veterans Affairs, and by small grants from the Office of the Vice President for Research and the Social and Behavioral Sciences Research Institute, University of Arizona. The research was also supported by National Science Foundation Grant BNS 9009619 to Stephen M. Kosslyn.

We thank Chris Azorson and Randy Buckner for comments and suggestions.

Correspondence concerning this article should be addressed to Chad J. Marsolek, Department of Psychology, University of Arizona, Tucson, Arizona 85721 . Electronic mail may be sent to marsolek@convx1.ccit.arizona.edu. under the conditions of Marsolek et al.'s (1992) study, visual cued recall of words is not greater when test cues are presented directly to the right hemisphere than when test cues are presented directly to the left hemisphere. In this article we examine a potential resolution to the apparently inconsistent results from PET and DVF studies of cued recall. We report a DVF experiment that uses the same procedure used in the PET experiment and show that, under these conditions, cued recall of words is greater when test cues are presented directly to the right hemisphere than when they are presented to the left. These results, in combination with the PET results, are consistent with the hypothesis that a visual form system, implemented at least in part in the right posterior cortex, can store information that supports implicit memory. This system may also interact with others to store information that underlies explicit memory.

In both Squire et al.'s (1992) PET experiment and Marsolek et al.'s (1992) DVF experiment, subjects completed visually presented word stems (three-letter beginnings of words; e.g., $C O N_{-}$) after judging how much they liked or disliked a list of visually presented words. When word-stem completion priming was tested, subjects were asked to complete the stems to form the first words that came to mind. Priming was measured as a greater tendency for subjects to produce words that they had recently read than words that they had not recently read. Word-stem completion priming is a form of implicit memory, which is exhibited when memory is expressed through a facilitation in performance of a cognitive task rather than through the recollection of information from a previous learning episode (Graf \& Schacter, 1985; Richardson-Klavehn \& Bjork, 1988; Roediger, 1990; Schacter, 1987, 1992a; Schacter, Chiu, \& Ochsner, 1993; Shimamura, 1986). In contrast, when wordstem cued recall was tested, subjects were asked to complete the word stems to form words that they had recently read. In this case the stems served as cues to aid in the recollection of words. Word-stem cued recall is a form of explicit memory (Graf \& Schacter, 1985). 
Results from both the PET and DVF studies indicate that processing in the right hemisphere produces more formspecific priming than processing in the left hemisphere. Such priming is obtained only when the words in the initial exposure phase and the subsequent word stems are presented in the same letter case. In the PET study, when subjects completed word stems to form the first words that came to mind and priming occurred, a region of posterior cortex in the right hemisphere was less activated than when priming could not occur because none of the possible word completions had been encountered during the initial exposure phase. In this study, all words and word stems were presented in the same letter case (uppercase). Similarly, in the DVF study, same-case priming (but not different-case priming) was greater when word stems were presented directly to the right hemisphere than to the left.

Both findings support the hypothesis that a form-specific system represents case-specific structural information and operates more effectively in the right hemisphere than in the left (see also Marsolek, in press; Marsolek \& Schacter, 1994). In contrast, an abstract visual form system represents word form identities in a more abstract manner and does not operate more effectively in the right hemisphere than in the left (Marsolek, in press). These are two of the perceptual systems that normally support visual priming (for others, see Schacter, 1990, 1992a, 1992b; Tulving \& Schacter, 1990).

However appealing this interpretation may seem for the priming results, it appears to fall short in the case of explicit memory because the results for explicit memory were not consistent in the PET and DVF studies. In the PET study, the hippocampal formation in the right hemisphere, but not in the left, was more active during the cued-recall task than during the priming task. It has been well established that the hippocampal formation plays an important role in explicit memory (e.g., Cohen \& Eichenbaum, 1993; Squire, 1987, 1992a, 1992b). Moreover, the finding that the right hippocampal formation was activated, but not the left, is consistent with the notion that the subjects in the PET experiment explicitly retrieved information stored in a formspecific system in the right hemisphere. This suggestion is plausible because in the PET study all words and word stems were presented in the same letter case (uppercase). In contrast, in the DVF study, same-case cued recall was not greater when stems were presented directly to the right hemisphere than to the left.

Differences in procedure between the PET and DVF studies may be responsible for this discrepancy in the results for cued recall. Three procedural differences between the studies are potentially important. First, in the PET experiment, all of the words and word stems were presented in the same letter case (uppercase). In the DVF experiment, only half of the word stems were presented in the same letter case as their corresponding words. Second, in the PET experiment, 15 words were presented during initial exposure, and 20 word stems were presented during cued recall. In contrast, in the DVF experiment, 45 words and 80 word stems were presented. Third, in the PET experiment, about $2 \mathrm{~min}$ intervened between the end of the initial exposure phase and the beginning of the cued-recall test, whereas approximately 6 min intervened in the DVF experiment.

We hypothesize that the procedure in the PET experiment led subjects to use the specific visual structure of the stems as a cue for recall, whereas the procedure in the DVF experiment did not. Under conditions in which rather few same-case-only stems were presented just a short time after the initial exposure of the target words, the specific visual structure of the stems could have been a very effective retrieval cue. Furthermore, under these conditions, cuedrecall performance ought to have been good. Indeed, subjects in the PET study correctly recalled $79 \%$ of the words, whereas subjects in the DVF study correctly recalled only $46 \%$ of the words. Hence, we suggest that subjects in the PET experiment were more likely to retrieve information from a form-specific system in the right hemisphere than were subjects in the DVF experiment.

\section{Experiment 1}

To test our hypothesis, we conducted a new DVF experiment to investigate same-case priming and cued recall. Subjects followed a procedure very much like that used in the PET experiment. If our hypothesis is correct, then we ought to find right-hemisphere superiority for same-case cued recall. The experiment also included baseline and priming tasks, which allowed us to attempt to replicate the earlier priming results that indicated right-hemisphere superiority for same-case priming (Marsolek et al., 1992).

\section{Method}

\section{Subjects}

Seventy-two University of Arizona undergraduate students volunteered to participate as subjects for course credit. All subjects were male, native English speakers, and right-handed. The mean laterality quotient was 0.72 , as assessed with the Edinburgh Handedness Inventory (Oldfield, 1971). Only right-handed men were tested in this and the following experiment because this group tends to exhibit greater hemispheric specialization of function than do women and left-handed men (e.g., Bradshaw \& Nettleton, 1983; Bryden, 1982; Kolb \& Whishaw, 1990; Springer \& Deutsch, 1981).

\section{Materials}

Eighty-five common English words of four to eight letters each served as stimulus materials. The mean word frequency was 70 per million (Kučera \& Francis, 1967). Each word began with a group of three letters (i.e., a stem) that was unique among the set of words and could be completed to form at least 10 common English words.

We created 12 lists of five words each to serve as experimental stimuli. These lists were balanced for word frequency, the number of common English words that began with the stem of each word, and the probability that a subject would generate the word when presented with its stem and asked to complete the stem with the first word that came to mind. Fifteen additional words were presented in the initial exposure phase of the baseline task for all 
subjects. The remaining 10 words were used as fillers. For both the priming and cued-recall tasks, three filler words were presented at the beginning and two filler words were presented at the end of each initial exposure list in order to attenuate primacy and recency effects.

A Macintosh II computer (Apple, Cupertino, CA) controlled the presentation of stimuli, which appeared on an AppleColor High Resolution RGB Monitor with a Polaroid (Norwood, MA) CP-50 filter placed over it to reduce glare. A chin rest was used to maintain a $50-\mathrm{cm}$ distance between the monitor and the eyes of the subjects. Black letters were presented against a white background on the monitor, in a 24-point, bold Helvetica font. Different letters of the alphabet varied in size but most were approximately $5 \times 6$ $\mathrm{mm}$, subtending $0.57^{\circ}$ of visual angle horizontally and $0.69^{\circ}$ of visual angle vertically. A central fixation point (a black dot) was used to indicate the beginning of each trial. The point was $2 \mathrm{~mm}$ in diameter and subtended $0.23^{\circ}$ of visual angle.

\section{Procedure}

Each subject was tested individually. The experiment contained three separate tasks: baseline, priming, and cued recall. The tasks were presented in that same order for all subjects. Within each task there was an exposure phase and a test phase. The exposure phase always took place before the test phase, and the test phase began about 2 min after the exposure phase ended. All variables were manipulated within subjects, and all of the words and word stems were always presented in uppercase letters. Thus, same-case memory was tested in this experiment.

In the exposure phases for all tasks, the subjects read 15 words, each presented in the central visual field. The subjects rated how much they liked or disliked each word on a 5-point scale $(1=$ dislike very much and $5=$ like very much). This task was designed to encourage semantic processing of each word. Each trial began with the appearance of the fixation point for $500 \mathrm{~ms}$ in the center of the monitor. Immediately after the fixation point disappeared, a word appeared for $3 \mathrm{~s}$ in the center of the monitor. The subject indicated his rating by pressing the appropriate number key on the keyboard. Each word remained on the screen for $3 \mathrm{~s}$, regardless of whether a number key was pressed before this time elapsed. The next trial began $1 \mathrm{~s}$ after the previous word disappeared from the monitor.

In the test phases for all tasks, each word stem was presented in the left or right visual field. Each trial began with the presentation of the fixation point in the center of the monitor for $500 \mathrm{~ms}$. Subjects were instructed to focus their eyes on the fixation point when it appeared. Immediately after the fixation point disappeared, a word stem appeared for $183 \mathrm{~ms}$ to the left or right of the center of the monitor. The center of the stem appeared $22 \mathrm{~mm}\left(2.5^{\circ}\right.$ of visual angle) from the center of the monitor, and the inner edge of any word stem never appeared closer than $13 \mathrm{~mm}\left(1.5^{\circ}\right.$ of visual angle) from the center of the monitor. A blank screen followed the disappearance of the word stem, and $3.32 \mathrm{~s}$ later the next trial began. Subjects were told that the experimenter recorded the words they produced.

Baseline task. In the exposure phase of the baseline task, all subjects rated the same list of 15 words, and they rated the set of 15 words twice in succession. In the test phase, the subjects were instructed that each of the word stems was the beginning of an English word and that their task was to add letters to each stem to form a common English word and then immediately speak it aloud. They were told to produce any English word but to report the first word that came to mind. They were also told not to produce proper nouns. In the baseline task, the word stems from four lists of 5 experimental words were presented to each subject. The word stems from two of these four lists appeared in the left visual field, whereas the word stems from the other two appeared in the right visual field. However, in the baseline task, none of the 20 word stems could have been completed to form words that had appeared during the exposure phase. The trials were presented in a different pseudorandom order for each subject. The orders were random with the constraint that no more than three word stems appeared consecutively in the same visual field.

Priming task. In the exposure phase of the priming and cuedrecall tasks, subjects rated two lists of 5 experimental words (plus 3 filler words presented before and 2 filler words presented after these 10 experimental words). As in the baseline task, the subjects rated the set of 15 words twice in succession. Different pseudorandom orders were used for each presentation of the 15 words for each task and for each subject. Each order was random with the constraint that no more than 3 words that had stems that would later be presented in the same visual field appeared consecutively.

In the test phase of the priming task, the word stems from four lists of 5 experimental words were presented to each subject. These four lists were not used in the baseline task. The procedure in this test phase was the same as that of the baseline test phase with the following exceptions. In the priming task, the word stems from two of the four lists were the stems of words that had appeared during the exposure phase (although subjects were not told about this). These were the target lists. The word stems from the other two lists were the stems of words that were not presented at any time in the experiment. These were the distractor lists. The word stems from one target list appeared in the left visual field, and those from the other, in the right visual field. The word stems from the distractor lists were similarly divided. Therefore, in the priming task, 10 word stems were targets and could have been completed to form words that had appeared in the immediately preceding exposure phase, and the other 10 word stems were distractors and could not have been completed to form previously presented words; five of the distractors and five of the targets appeared in the left visual field, and the other five of the distractors and targets appeared in the right visual field. The trials were presented in a different pseudorandom order for each subject. The orders were random with the constraints that no more than 3 target word stems appeared consecutively, no more than 3 distractor word stems appeared consecutively, and no more than 3 word stems appeared consecutively in the same visual field.

Cued-recall task. The exposure and test phases of the cuedrecall task were conducted in the same manner as in the priming task with the following exception. In the cued-recall test phase, the subjects were instructed to try to recall words they had seen in the exposure phase in order to complete the word stems. The subjects were encouraged to guess when they could not recall a word from the like-dislike rating task. They were also told that only some of the word stems could be completed to form words from the like-dislike task.

The 12 lists of experimental words were rotated through the three types of tasks, the two levels of the target-distractor condition, and the two visual fields of word-stem presentation, so that each five-word list appeared in all conditions an equal number of times.

\section{Results}

In this and the following experiment, we used a strict criterion to determine whether a response word was one of the 60 words from the 12 experimental word lists (critical words). The response for a trial was scored as a critical word 
only if it was exactly the same as the critical word associated with the word stem presented in that trial. Thus, no plural forms, past tense forms, or other changes from an original critical word were accepted. Separate repeated measures analyses of variance (ANOVAs) were conducted for the word-stem completion results (including both baseline and priming) and for the cued-recall results. ${ }^{1}$

\section{Word Completion}

Figure 1 depicts the results from the word-stem completion tasks. In the analysis of these results, critical-word completion rate (for all items in the baseline task and for target items in the priming task) was the dependent variable. Test task (baseline vs. priming) and test hemisphere (left vs. right) were within-subjects independent variables.

A main effect of test task was found. Completion rates were greater in the priming task $(65.3 \%)$ than in the baseline task $(10.0 \%), F(1,71)=429, p<.0001$, MSe $=512.7$, which indicates that significant priming was obtained. Furthermore, completion rates were greater when stems were presented directly to the right hemisphere $(41.5 \%)$ than to the left $(33.8 \%), F(1,71)=15.8, p<.001, \mathrm{MSe}=272.2$, for the main effect of test hemisphere.

More important, the interaction between test task and test hemisphere was significant, $F(1,71)=7.00, p<$ $.01, \mathrm{MSe}=241.4$. This interaction indicates that the overall right-hemisphere advantage in completion rates re-

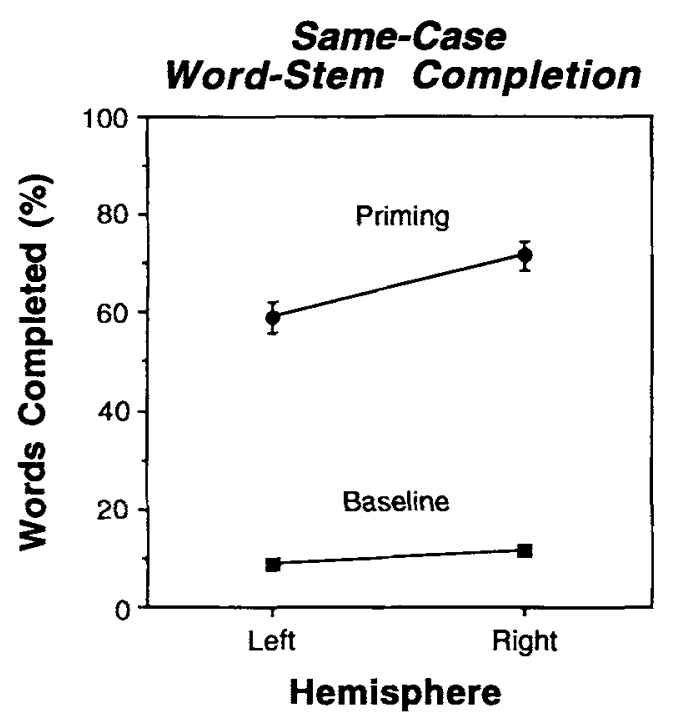

Figure 1. Results from the baseline and priming word-stem completion tasks of Experiment 1, in which same-case implicit memory was examined. (Mean critical-word completion rates for all trials in the baseline task and for target trials in the priming task are presented as a function of test task [baseline vs. priming] and test hemisphere [left vs. right]. Baseline reflects critical-word completion rates when critical words were not previously presented, and priming reflects critical-word completion rates when the stems could be completed to form previously presented critical words. Error bars indicate standard errors of the mean.) flected rates in the priming task more than rates in the baseline task. In fact, completion rates in the priming task were greater when stems were presented directly to the right hemisphere $(71.6 \%)$ than to the left $(59.0 \%), F(1$, $142)=22.1, p<.0001$, MSe $=256.8$, for the simple effect, whereas completion rates in the baseline task were not greater when stems were presented directly to the right hemisphere $(11.5 \%)$ than to the left $(8.6 \%), F(1$, $142)=1.16, p>.25$, MSe $=256.8$, for the simple effect. These results demonstrate that a right-hemisphere advantage was found in priming performance.

\section{Cued Recall}

The results from the cued-recall task are shown in Figure 2. In the analysis of these results, critical-word completion rate was the dependent variable. Test stimulus (distractor vs. target) and test hemisphere (left vs. right) were withinsubjects independent variables.

In a main effect of test stimulus, completion rates for target stems $(76.8 \%)$ were greater than completion rates for distractor stems $(10.7 \%), F(1,71)=763, p<.0001$, $\mathrm{MSe}=412.0$, which indicates significant explicit-memory performance. The main effect of test hemisphere did not approach significance, $F<1.00$.

Most important, the interaction between test stimuli and test hemisphere was significant, $F(1,71)=5.93, p<.05$, $\mathrm{MSe}=328.6$, which indicates a right-hemisphere advantage for explicit-memory performance. Indeed, simple effects revealed that completion rates for target stems were greater when they were presented directly to the right hemisphere $(80.4 \%)$ than to the left $(73.3 \%), F(1,142)=5.72$, $p<.05, \mathrm{MSe}=319.5$; whereas completion rates for distractor stems did not differ whether stems were presented directly to the right hemisphere $(9.1 \%)$ or to the left $(12.8 \%), F(1,142)=1.21, p>.25, \mathrm{MSe}=319.5$.

\section{Discussion}

As expected, the word completion results replicate a key finding from the earlier DVF priming study (Marsolek et al., 1992). In that study, Marsolek et al. found a right-hemisphere advantage for same-case priming. In this experiment all items were presented in the same letter case, and we found a right-hemisphere priming advantage. We expected these results because we hypothesized that a form-specific

\footnotetext{
${ }^{1}$ In the analyses in Experiments 1 and 2, the percentages calculated for the dependent measures were conditionalized, in that only trials in which the word stems were accurately identified by the subjects were included in the analyses. The analyses of conditionalized percentages did not differ qualitatively from analyses of nonconditionalized percentages, largely because subjects identified the word stems accurately in a high proportion of the trials (93\%). In addition, we could not perform valid analyses of response times for critical-word completions because a large number of cells in the design did not include an observation in which a critical word was produced.
} 


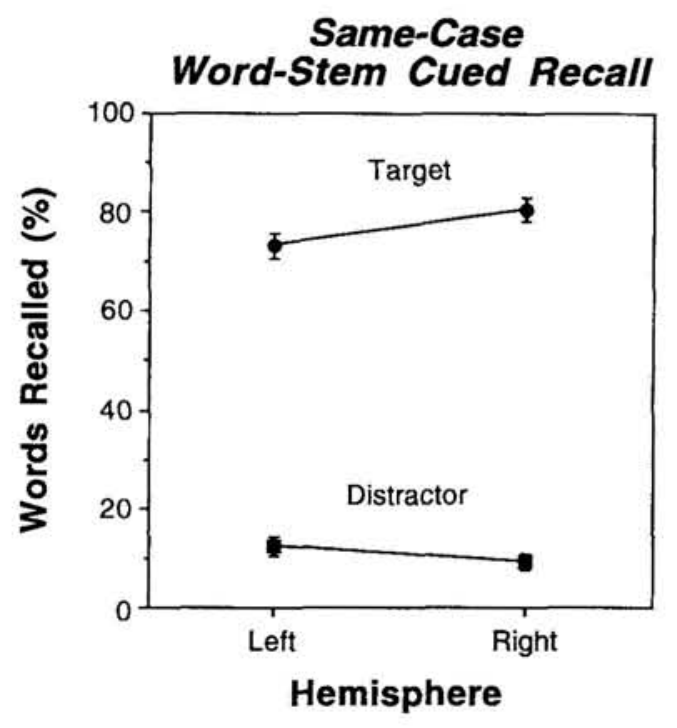

Figure 2. Results from the cued-recall task in Experiment 1, in which same-case explicit memory was examined. (Mean criticalword completion rates are presented as a function of test stimulus [distractor vs. target] and test hemisphere [left vs. right]. Distractor rates reflect critical-word completions when critical words were not previously presented, and target rates reflect correct recall of previously presented critical words when their stems were presented as cues. Error bars indicate standard errors of the mean.)

system underlies same-case priming and operates more effectively in the right hemisphere than in the left.

Also as predicted, the cued-recall results contrast with a finding from the earlier DVF study. In that study there was no right-hemisphere advantage for same-case cued recall (Marsolek et al., 1992). However, in this experiment, all items were presented in the same letter case, and we found a right-hemisphere advantage in cued recall. We hypothesized that the procedure in this experiment encouraged subjects to retrieve information from a form-specific system in the right hemisphere during cued recall, which Marsolek et al.'s procedure did not.

The results from this experiment are important because they corroborate findings from the earlier PET study (Squire et al., 1992) that used the same procedure as the one used in this DVF experiment. In the PET study, same-case implicit and explicit memory were associated with activation changes in the right hemisphere. It is important to note that additional PET experiments have been conducted more recently (Buckner et al., in press). The recent PET experiments have also used a similar procedure and have shown that different-case implicit and explicit memory produced different patterns of results from same-case implicit and explicit memory. First, different-case priming produced an activation change that was localized to a different region of right posterior cortex compared with same-case priming. Second, different-case cued recall did not activate the right hippocampal region. These findings are consistent with the hypotheses that a form-specific system in the right hemisphere operates independently of an abstract visual form system and stores information that is retrieved during samecase cued recall, but not during different-case cued recall, under the conditions of the PET experiments. One way to further confirm this hypothesis is to investigate differentcase memory in another DVF experiment that also follows the PET procedure.

\section{Experiment 2}

Experiment 2 was conducted in the same manner as Experiment 1 with one exception. Following the procedure from the recent PET study (Buckner et al., in press), we presented all initial-exposure words in lowercase letters. When all test stems were then presented in uppercase letters, different-case memory was tested. If our hypotheses are correct, then we ought not to find right-hemisphere superiority for different-case priming or different-case cued recall.

\section{Method}

\section{Subjects}

Seventy-two University of Arizona undergraduate students volunteered to participate as subjects for course credit. All subjects were male, native English speakers, and right-handed. The mean laterality quotient was 0.71 , as assessed with the Edinburgh Handedness Inventory (Oldfield, 1971). None of the subjects in this experiment participated in Experiment 1.

\section{Materials and Procedure}

The materials and procedure in this experiment were the same as in Experiment 1 with the exception that all words in the exposure phases were presented in lowercase letters. Word stems in the test phases were always presented in uppercase letters (as in Experiment 1), and thus, different-case memory was tested in this experiment.

\section{Results}

As in Experiment 1, separate repeated measures ANOVAs were conducted for the word-stem completion results (including both baseline and priming) and for the cued-recall results. One final repeated measures ANOVA was conducted for the combined results from Experiments 1 and 2 .

\section{Word Completion}

The results from the word-stem completion tasks are shown in Figure 3. Critical-word completion rate (for all items in the baseline task and for target items in the priming task) was the dependent variable, and test task (baseline vs. priming) and test hemisphere (left vs. right) were withinsubjects independent variables in this analysis.

Completion rates were greater in the priming task $(56.5 \%)$ than in the baseline task $(9.6 \%), F(1,71)=321$, $p<.0001, \mathrm{MSe}=492.7$, for the main effect of test task, which indicates that significant priming was obtained. The 


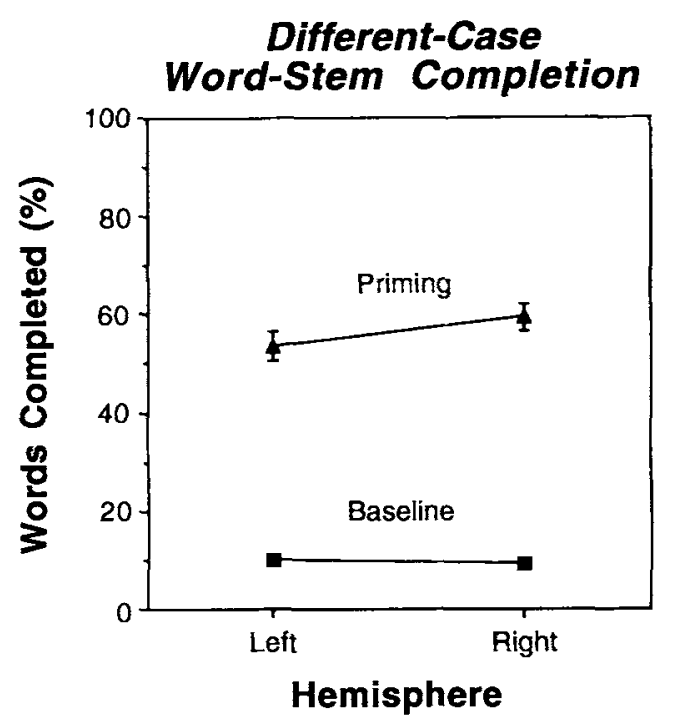

Figure 3. Results from the baseline and priming word-stem completion tasks of Experiment 2, in which different-case implicit memory was examined. (Mean critical-word completion rates for all trials in the baseline task and for target trials in the priming task are presented as a function of test task [baseline vs. priming] and test hemisphere [left vs. right]. Baseline reflects critical-word completion rates when critical words were not previously presented, and priming reflects critical-word completion rates when the stems could be completed to form previously presented critical words. Error bars indicate standard errors of the mean.)

main effect of test hemisphere was not significant, $F(1$, $71)=2.30, p>.13, \mathrm{MSe}=222.3$. More important, as predicted, the interaction between test task and test hemisphere was not significant, $F(1,71)=2.69, p>.10$, MSe $=261.8$. Thus, no hemispheric advantage was found in priming performance.

\section{Cued Recall}

Figure 4 illustrates the results from the cued-recall task. Critical-word completion rate was the dependent variable, and test stimulus (distractor vs. target) and test hemisphere (left vs. right) were within-subjects independent variables in this analysis.

In a main effect of test stimulus, the completion rate for target stems $(63.5 \%)$ was greater than the completion rate for distractor stems $(10.9 \%), F(1,71)=418, p<.0001$, $\mathrm{MSe}=477.2$, which indicates significant explicit-memory performance. Furthermore, completion rates were greater when stems were presented directly to the right hemisphere $(39.7 \%)$ than to the left $(34.6 \%), F(1,71)=6.22, p<.05$, MSe $=300.8$, for the main effect of test hemisphere. Most important, however, the interaction between test stimuli and test hemisphere did not approach significance $(F<1.00)$, which indicates that no hemispheric advantage was found for explicit-memory performance.

\section{Priming and Cued-Recall Difference Scores}

Although the results from this experiment do not demonstrate a right-hemisphere advantage for different-case priming or different-case cued recall, there were trends for right-hemisphere superiority (see Figures 3 and 4). Thus, we felt it was prudent to compare directly same-case and different-case memory performance in Experiments 1 and 2. We combined the results from both experiments and reanalyzed them in terms of difference scores (see Figure 5). The left side of Figure 5 shows the priming difference scores. To calculate these scores, for each subject, we subtracted the critical-word completion rate for left-hemisphere trials in the baseline task from the rate for left-hemisphere target trials in the priming task, and we did the same for right-hemisphere trials. The right side of Figure 5 illustrates the cued-recall difference scores. To calculate these scores, for each subject, we subtracted the critical-word completion rate for left-hemisphere distractor trials from the rate for left-hemisphere target trials in the cued-recall task, and we did the same for right-hemisphere trials. In this analysis, difference score was the dependent variable. Letter case of previous word presentation (same case vs. different case) was a between-subjects independent variable, and form of memory (priming vs. cued recall) and test hemisphere (left vs. right) were within-subjects independent variables.

Same-case memory $(60.7 \%)$ was greater than differentcase memory (49.8\%), as indicated by a main effect of letter case, $F(1,142)=13.6, p<.001, \mathrm{MSe}=1265.7$. In addi-

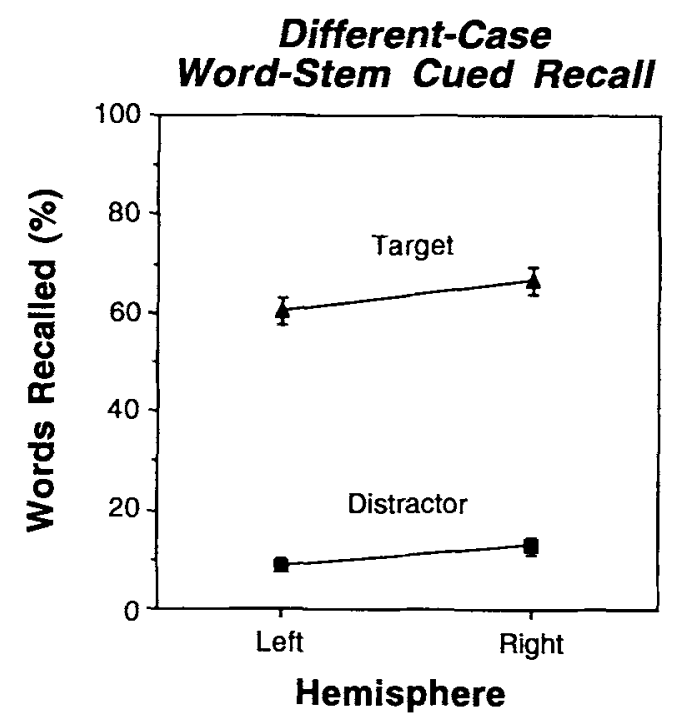

Figure 4. Results from the cued-recall task in Experiment 2, in which different-case explicit memory was examined. (Mean critical-word completion rates are presented as a function of test stimulus [distractor vs. target] and test hemisphere [left vs. right]. Distractor rates reflect critical-word completions when critical words were not previously presented, and target rates reflect correct recall of previously presented critical words when their stems were presented as cues. Error bars indicate standard errors of the mean.) 


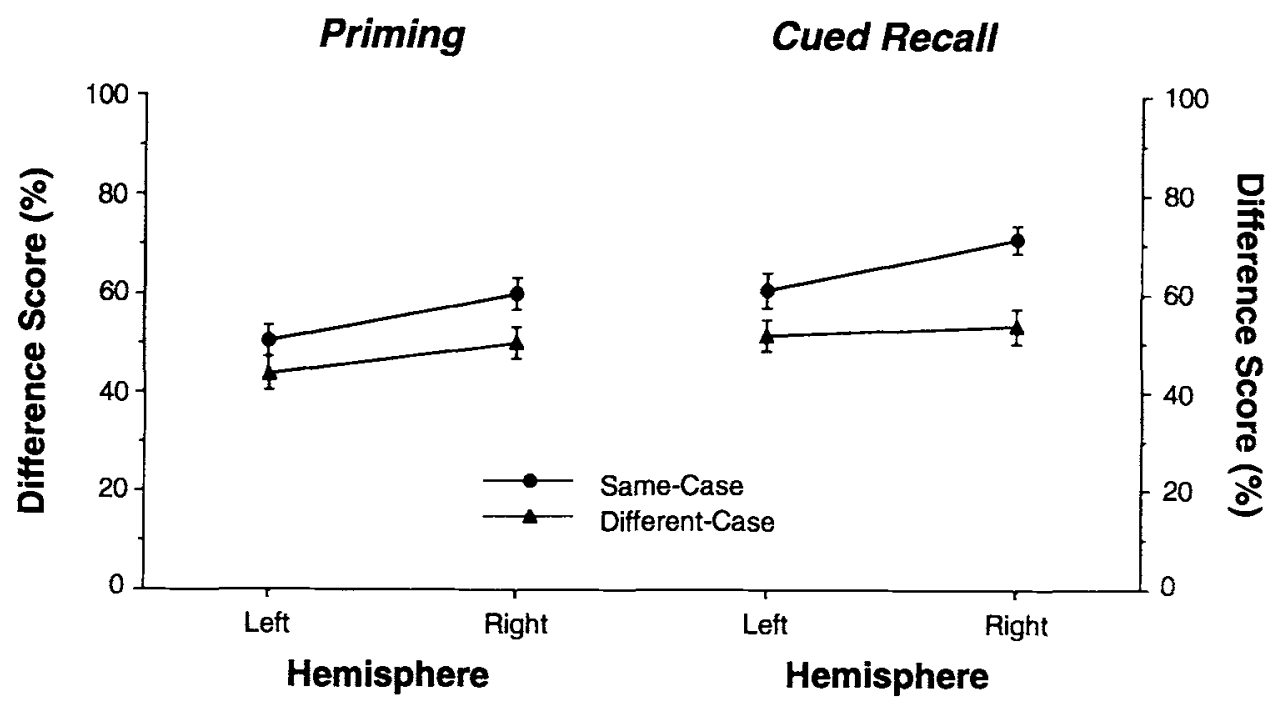

Figure 5. Combined results from both Experiments 1 and 2. (Mean difference scores [for priming scores, critical-word completion rates for target trials in the priming task less critical-word completion rates for all trials in the baseline task; for cued-recall scores, critical-word completion rates for target trials less critical-word completion rates for distractor trials] are presented as a function of letter case of previously presented words [same case vs. different case] and test hemisphere [left vs. right]. Error bars indicate standard errors of the mean.)

tion, cued-recall performance $(59.4 \%)$ was greater than priming performance $(51.1 \%), F(1,142)=15.6, p<.0001$, $\mathrm{MSe}=628.9$, for the main effect of form of memory. Furthermore, memory performance was greater when stems were presented directly to the right hemisphere $(58.8 \%)$ than to the left $(51.7 \%), F(1,142)=13.8, p<.001$, $\mathrm{MSe}=523.8$, for the main effect of test hemisphere. None of the interactions in this analysis were significant $(p s>.10)$. In particular, the interaction between letter case and test hemisphere was not significant, $F(1,142)=$ $2.42, p>.10, \mathrm{MSe}=523.8$.

However, in order to test planned contrasts derived from the hypothesis and from the reported analyses, we tested the following simple effects. As predicted, a significant simple effect revealed that same-case priming was greater when stems were presented directly to the right hemisphere $(60.1 \%)$ than to the left $(50.4 \%), F(1,284)=5.99, p<.05$, $\mathrm{MSe}=564.2$, for the simple effect. In contrast, differentcase priming was not greater when stems were presented directly to the right hemisphere $(50.0 \%)$ than to the left $(43.8 \%), F(1,284)=2.50, p>.10, \mathrm{MSe}=564.2$, for the simple effect. Similarly, same-case cued recall was greater when stems were presented directly to the right hemisphere $(71.3 \%)$ than to the left $(60.9 \%), F(1,284)=6.91, p<.01$, $\mathrm{MSe}=564.2$, for the simple effect. In contrast, differentcase cued recall was not greater when stems were presented directly to the right hemisphere $(53.6 \%)$ than to the left $(51.6 \% ; F<1.00$, for the simple effect).

\section{Discussion}

As predicted, the results from this experiment contrast with those from Experiment 1. Unlike same-case priming in the previous experiment, different-case priming in this experiment did not reveal a significant right-hemisphere advantage. The results from this experiment replicate another important finding from Marsolek et al.'s (1992) DVF priming study, in which they did not find a right-hemisphere advantage for different-case priming. Therefore, we suggest that a form-specific system in the right hemisphere underlies same-case priming but does not substantially underlie different-case priming.

Also as expected, different-case cued recall in this experiment did not reveal a right-hemisphere advantage, in contrast with same-case cued recall in Experiment 1. We expected this difference between experiments because we hypothesized that the procedure in Experiment 1, but not the procedure in Experiment 2 or in the earlier DVF study, encouraged subjects to retrieve information from a formspecific system in the right hemisphere during cued recall.

One surprising result from this experiment was the significant main effect of test hemisphere during the cuedrecall task (targets and distractors combined; see Figure 4). Although there was no interaction between test stimulus and test hemisphere (i.e., no right-hemisphere advantage for different-case cued recall), there was a general right-hemisphere advantage in completion rates for all items. In other words, subjects produced a greater number of critical-word completions (both correct retrievals for targets and correct guesses for distractors) when stems were presented directly to the right hemisphere than to the left. We did not predict this result, but one may suspect that a conceptualassociative system in the right hemisphere contributed to the effect. This may happen if subjects generate many word completions for each stem and select one for response during cued recall, and if subjects activate a greater number 
and wider range of word meanings in response to an input to the right hemisphere than to the left (see Beeman et al., 1994; Burgess \& Simpson, 1988; Chiarello, 1991). If so, a right-hemisphere conceptual-associative system may generate a greater number of possible word completions and hence may help to make both correct retrievals and correct guesses more often than a left-hemisphere conceptualassociative system. The greater the number of candidates generated, the greater the chance of hitting (i.e., correctly recalling when a target stem is presented) or choosing (i.e., correctly guessing when a distractor stem is presented) one of the critical words, all of which are distinctively mediumfrequency, commonplace words. This explanation is very speculative, however, and we admit that the result may be spurious. Indeed, the effect was rather small $(5.1 \% ; 39.7 \%$ for right-hemisphere presentations compared with $34.6 \%$ for left-hemisphere presentations).

\section{General Discussion}

The results from these DVF experiments converge with findings from previous PET studies (Buckner et al., in press; Squire et al., 1992). In these DVF experiments, which used the procedure from the PET studies, both same-case priming and same-case cued recall were greater when word stems were presented directly to the right hemisphere than when stems were presented to the left. In contrast, different-case priming and different-case cued recall were not greater when stems were presented directly to the right hemisphere as compared with the left. These results corroborate the main findings from the PET studies, in which activation in the right hippocampal formation was associated with samecase cued recall, but not different-case cued recall, and changes in activity in a particular region of the right posterior cortex were associated with same-case priming, but not different-case priming. ${ }^{2}$ Therefore, when a similar procedure is used, both the DVF and PET experiments support three hypotheses: (a) A form-specific system operates more effectively in the right hemisphere than in the left, (b) this system supports same-case implicit memory, and (c) this system may also interact with other systems to support same-case explicit memory.

In both this DVF study and the previous one reported by Marsolek et al. (1992), same-case priming was greater when word stems were presented directly to the right hemisphere than to the left. However, there were differences in the cued-recall findings from the two DVF studies. In this study same-case cued recall was greater when stems were presented directly to the right hemisphere than to the left. This result was not obtained in Marsolek et al.'s study. We propose that the cued-recall procedure that we used for the DVF experiments, but which was not used in the previous DVF study, encouraged subjects to retrieve information from a form-specific system in the right hemisphere. Because this DVF study used the same procedure that was used in the PET study, this reasoning also explains why same-case cued recall produced activation in the right hemisphere in the PET study.
This reasoning may also help to explain findings from another PET experiment and from previous cognitive studies of memory. First, in Sergent, Ohta, and MacDonald's (1992) PET study, the right parahippocampal gyrus (but not the left) was activated when subjects classified famous faces as actors or nonactors, after all of the faces had been shown to subjects the day before. To the extent that memory for the previous day's exposure to each face influenced processing in this task, subjects may have retrieved recently stored form-specific information in a right-hemisphere system during the classification task. Second, past cognitive studies of memory have typically revealed only small, nonsignificant effects of case or font on explicit memory (Blaxton, 1989; Roediger \& Blaxton, 1987; but for a significant effect of case, see Kirsner, 1973). These results may have been obtained because the procedures in these studies are generally more similar to the procedure in Marsolek et al.'s (1992) DVF study than to the procedure in this DVF study or Squire et al.'s (1992) PET study, in which subjects apparently were encouraged to retrieve information from a form-specific system.

Our findings also rule out an alternative explanation for the different results obtained for same-case cued recall in the PET study and in the previous DVF study. The alternative rests on the fact that the visual field of word-stem presentation was different in these studies. Each stem in the PET experiment was presented in the central visual field for $3 \mathrm{~s}$, whereas each stem in the DVF experiments was presented in the left or right visual field for $183 \mathrm{~ms}$. One may suppose that bilateral stimulation from centrally presented same-case stems always produces stronger activation and more efficient processing in the right hemisphere than in the left, whereas unilateral stimulation from same-case stems in the left or right visual fields produces stronger activation and more efficient processing in the contralateral hemisphere than in the ipsilateral hemisphere. If so, then the right-hemisphere hippocampal activation during same-case cued recall in the PET experiment, and the lack of a hemispheric advantage for same-case cued recall in the previous DVF study, may be explained without reference to a formspecific system. However, our findings do not support this hypothesis because in the present DVF study we found a right-hemisphere advantage for same-case cued recall.

\footnotetext{
${ }^{2}$ More specifically, changes in activity in right posterior cortex were localized to two slightly different regions for same-case and different-case priming, whereas changes in activity in left posterior cortex were localized to the same region for same-case and different-case priming (Buckner et al., in press). In addition, changes in right posterior cortex were numerically larger than changes in left posterior cortex in different-case priming, as well as in samecase priming. This pattern of results may be consistent with the nonsignificant trend toward a right-hemisphere advantage for different-case priming, as well as the significant right-hemisphere advantage for same-case priming in this DVF study (see Figure 5). More important, though, the separate regions of changes in activity in the same-case and different-case PET conditions suggest that a form-specific system and an abstract visual form system operate independently.
} 
The results reported in this article provide further support for the hypothesis that a form-specific system operates more effectively in the right hemisphere than in the left. This system appears to be one of several that store perceptual, conceptual, or motoric information in neocortical regions of the brain (e.g., Gabrieli, Milberg, Keane, \& Corkin, 1990; Kosslyn, 1987; Kosslyn, Flynn, Amsterdam, \& Wang, 1990; Schacter, 1990, 1992a, 1992b; Squire, 1987, 1992a; Tulving \& Schacter, 1990; Ungerleider \& Mishkin, 1982). For a brief time after such a system produces a particular output in response to receiving a particular input, that inputoutput mapping (or a close approximation, as in primed word-stem completion) will take place more efficiently than if the prime event had not taken place. This facilitation may account for repetition priming and for many implicit memory effects. An important aspect of this mechanism is that processing in the hippocampal formation is not necessary for these effects (e.g., Cohen \& Eichenbaum, 1993; Squire, 1987, 1992a, 1992b).

In contrast, the hippocampal formation and other medial temporal lobe and diencephalic brain structures are necessary for explicit memory. Processing in these systems interacts with neocortical systems in order to store links between to-be-remembered information and novel elements of the encoding situation (e.g., time and place context). Such links are important for storage and retrieval processes that subserve explicit memory for unconsolidated information (newly learned information that requires an intact hippocampal system for storage and retrieval until it has become consolidated in memory; e.g., Squire, 1987). In this framework, to-be-remembered information and information about novel aspects of the encoding situation are stored in neocortical systems. However, interactions between processing in the hippocampal formation and processing in the neocortical systems are needed to create the functional links that help to support unconsolidated explicit memory. This framework provides a way to explain how a form-specific system can be involved in both same-case explicit memory and same-case implicit memory.

One way to understand the results reported in this article is that a system that operates more effectively in the right hemisphere than in the left stores form-specific information that contributes to both explicit memory and implicit memory. Although these results do not identify which brain regions in the right hemisphere store form-specific information, the combination of these results and the PET results (as well as evidence from other sources; see Marsolek et al., 1992) suggests a possibility. The posterior cortex of the right hemisphere implements a form-specific system, which can support same-case implicit memory through repeated processing of form-specific information. Furthermore, the hippocampal formation of the right hemisphere may interact with this system, as well as with other neocortical systems, to subserve explicit memory for form-specific information.

\section{References}

Beeman, M., Friedman, R. B., Grafman, J., Perez, E., Diamond, S., \& Lindsay, M. B. (1994). Summation priming and coarse coding in the right hemisphere. Journal of Cognitive Neuroscience, 6 , $26-45$.

Blaxton, T. A. (1989). Investigating dissociations among memory measures: Support for a transfer appropriate processing framework. Journal of Experimental Psychology: Learning, Memory, and Cognition, 15, 657-668.

Bradshaw, J. L., \& Nettleton, N. C. (1983). Human cerebral asymmetry. Englewood Cliffs, NJ: Prentice-Hall.

Bryden, M. P. (1982). Laterality: Functional asymmetry in the intact brain. San Diego, CA: Academic Press.

Buckner, R. L., Petersen, S. E., Ojemann, J. G., Miezin, F. M., Squire, L. R., \& Raichle, M. E. (in press). Functional anatomical studies of explicit and implicit memory retrieval tasks. Joumal of Neuroscience.

Burgess, C., \& Simpson, G. (1988). Cerebral hemispheric mechanisms in the retrieval of ambiguous word meanings. Brain and Language, 33, 86-103.

Chiarello, C. (1991). Interpretation of word meanings by the cerebral hemispheres: One is not enough. In P. J. Schwanenflugel (Ed.), The psychology of word meanings (pp. 251-278). Hillsdale, NJ: Erlbaum.

Cohen, N. J., \& Eichenbaum, H. (1993). Memory, amnesia, and the hippocampal system. Cambridge, MA: MIT Press.

Gabrieli, J. D. E., Milberg, W., Keane, M. M., \& Corkin, S. (1990). Intact priming of patterns despite impaired memory. Neuropsychologia, 28, 417-428.

Graf, P., \& Schacter, D. L. (1985). Implicit and explicit memory for new associations in normal and amnesic subjects. Journal of Experimental Psychology: Learning, Memory, and Cognition, 11, 501-518.

Kirsner, K. (1973). An analysis of the visual component in recognition memory for verbal stimuli. Memory \& Cognition, $l$, 449-453.

Kolb, B, \& Wishaw, I. Q. (1990). Fundamentals of human neuropsychology. San Francisco: Freeman.

Kosslyn, S. M. (1987). Seeing and imagining in the cerebral hemispheres: A computational approach. Psychological Review, 94, 148-175.

Kosslyn, S. M., Flynn, R. A., Amsterdam, J. B., \& Wang, G. (1990). Components of high-level vision: A cognitive neuroscience analysis and accounts of neurological syndromes. Cognition, 34, 203-277.

Kučera, M., \& Francis, W. (1967). Computational analysis of present-day American English. Providence, RI: Brown University Press.

Marsolek, C. J. (in press). Abstract visual form representations in the left cerebral hemisphere. Journal of Experimental Psychology: Human Perception and Performance.

Marsolek, C. J., Kosslyn, S. M., \& Squire L. R. (1992). Formspecific visual priming in the right cerebral hemisphere. Journal of Experimental Psychology: Leaming, Memory, and Cognition, 18, 492-508.

Marsolek, C. J., \& Schacter, D. L. (1994). Form-specific visual priming for new associations in the right cerebral hemisphere. Manuscript submitted for publication.

Oldfield, R. C. (1971). The assessment and analysis of handedness: The Edinburgh Inventory. Neuropsychologia, 9, 97-113.

Petersen, S. E., \& Fiez, J. A. (1993). The processing of single words studied with positron emission tomography. Annual Review of Neuroscience, 16, 509-530.

Richardson-Klavehn, A., \& Bjork, R. A. (1988). Measures of memory. Annual Review of Psychology, 36, 475-543.

Roediger, H. L., III. (1990). Implicit memory: Retention without remembering. American Psychologist, 45, 1043-1056. 
Roediger, H. L., III, \& Blaxton, T. A. (1987). Retrieval modes produce dissociations in memory for surface information. In $\mathrm{D}$. S. Gorfein \& R. R. Hoffman (Eds.), Memory and cognitive processes: The Ebbinghaus Centennial Conference (pp. 349379). Hillsdale, NJ: Erlbaum.

Schacter, D. L. (1987). Implicit memory: History and current status. Journal of Experimental Psychology: Learning, Memory, and Cognition, 13, 501-518.

Schacter, D. L. (1990). Perceptual representation systems and implicit memory: Toward a resolution of the multiple memory systems debate. In A. Diamond (Ed.), Annals of the New York Academy of Sciences: Vol. 608. The development and neural bases of higher cognitive functions (pp. 543-571). New York: New York Academy of Sciences.

Schacter, D. L. (1992a). Priming and multiple memory systems: Perceptual mechanisms of implicit memory. Joumal of Cognitive Neuroscience, 4, 244-256.

Schacter, D. L. (1992b). Understanding implicit memory: A cognitive neuroscience approach. American Psychologist, 47, 559569.

Schacter, D. L., Chiu, C.-Y. P., \& Ochsner, K. N. (1993). Implicit memory: A selective review. Annual Review of Neuroscience, $16,159-182$.

Sergent, J., Ohta, S., \& MacDonald, B. (1992). Functional neuroanatomy of face and object processing. Brain, 115, 15-36.

Shimamura, A. P. (1986). Priming effects in amnesia: Evidence for a dissociable memory function. The Quarterly Journal of Ex- perimental Psychology, 38A, 619-644.

Springer, S. P., \& Deutsch, G. (1981). Left brain, right brain. San Francisco: Freeman.

Squire, L. R. (1987). Memory and brain. New York: Oxford University Press.

Squire, L. R. (1992a). Declarative and nondeclarative memory: Multiple brain systems supporting learning and memory. Journal of Cognitive Neuroscience, 4, 232-243.

Squire, L. R. (1992b). Memory and the hippocampus: A synthesis from findings with rats, monkeys, and humans. Psychological Review, 99, 195-231.

Squire, L. R., Ojemann, J. G., Miezin, F. M., Petersen, S. E., Videen, T. O., \& Raichle, M. E. (1992). Activation of the hippocampus in humans: A functional anatomical study of memory. Proceedings of the National Academy of Sciences of the United States of America, 89, 1837-1841.

Tulving, E., \& Schacter, D. L. (1990). Priming and human memory systems. Science, 247, 301-305.

Ungerleider, L. G. \& Mishkin, M. (1982). Two cortical visual systems. In D. J. Ingle, M. A. Goodale, \& R. J. W. Mansfield (Eds.), The analysis of visual behavior (pp. 549-586). Cambridge, MA: MIT Press.

Received October 5, 1993

Revision received March 14, 1994

Accepted March 16, 1994

\section{New Editors Appointed, 1996-2001}

The Publications and Communications Board of the American Psychological Association announces the appointment of two new editors for 6-year terms beginning in 1996. As of January 1, 1995, manuscripts should be directed as follows:

- For Behavioral Neuroscience, submit manuscripts to Michela Gallagher, $\mathrm{PhD}$, Department of Psychology, Davie Hall, CB\# 3270, University of North Carolina, Chapel Hill, NC 27599.

- For the Joumal of Experimental Psychology: Learning, Memory, and Cognition, submit manuscripts to James H. Neely, PhD, Editor, Department of Psychology, State University of New York at Albany, 1400 Washington Avenue, Albany, NY 12222.

Manuscript submission patterns make the precise date of completion of 1995 volumes uncertain. The current editors, Larry R. Squire, $\mathrm{PhD}$, and Keith Rayner, $\mathrm{PhD}$, respectively, will receive and consider manuscripts until December 1994. Should either volume be completed before that date, manuscripts will be redirected to the new editors for consideration in 1996 volumes. 